\title{
Dauer signalling pathway model for Haemonchus contortus
}

\author{
Guangxu Ma, Tao Wang, Pasi K. Korhonen, Andreas J. Stroehlein, Neil D. Young and Robin B. Gasser*
}

\begin{abstract}
Background: Signalling pathways have been extensively investigated in the free-living nematode Caenorhabditis elegans, but very little is known about these pathways in parasitic nematodes. Here, we constructed a model for the dauer-associated signalling pathways in an economically highly significant parasitic worm, Haemonchus contortus.

Methods: Guided by data and information available for C. elegans, we used extensive genomic and transcriptomic datasets to infer gene homologues in the dauer-associated pathways, explore developmental transcriptomic, proteomic and phosphoproteomic profiles in H. contortus and study selected molecular structures.

Results: The canonical cyclic guanosine monophosphate (cGMP), transforming growth factor- $\beta$ (TGF- $\beta$ ), insulin-like growth factor 1 (IGF-1) and steroid hormone signalling pathways of $\mathrm{H}$. contortus were inferred to represent a total of 61 gene homologues. Compared with C. elegans, H. contortus has a reduced set of genes encoding insulin-like peptides, implying evolutionary and biological divergences between the parasitic and free-living nematodes. Similar transcription profiles were found for all gene homologues between the infective stage of $\mathrm{H}$. contortus and dauer stage of $C$. elegans. High transcriptional levels for genes encoding G protein-coupled receptors (GPCRs), TGF- $\beta$, insulin-like ligands (e.g. ins-1, ins-17 and ins-18) and transcriptional factors (e.g. daf-16) in the infective L3 stage of H. contortus were suggestive of critical functional roles in this stage. Conspicuous protein expression patterns and extensive phosphorylation of some components of these pathways suggested marked post-translational modifications also in the L3 stage. The high structural similarity in the DAF-12 ligand binding domain among nematodes indicated functional conservation in steroid (i.e. dafachronic acid) signalling linked to worm development.
\end{abstract}

Conclusions: Taken together, this pathway model provides a basis to explore hypotheses regarding biological processes and regulatory mechanisms (via particular microRNAs, phosphorylation events and/or lipids) associated with the development of H. contortus and related nematodes as well as parasite-host cross talk, which could aid the discovery of new therapeutic targets.

Keywords: Haemonchus contortus, Dauer signalling pathway, Transcriptomic analysis, Proteomic analysis, Phosphoproteomic analysis

\section{Background}

Signal transduction is a process by which physical or chemical impulses are transmitted through a cell via a series of molecular events $[1,2]$. Many signalling pathways involve the binding of molecules (called ligands) to receptors that initiate or activate processes within cells. Usually, ligand-binding leads to conformational

*Correspondence: robinbg@unimelb.edu.au

Department of Veterinary Biosciences, Melbourne Veterinary School, The University of Melbourne, Parkville, VIC 3010, Australia alteration(s) in a receptor, which triggers a chain of biochemical events that regulate processes such as transcription and translation, post-translational or conformational alterations of proteins and/or the location of expression [3-5].

Signalling pathways have been extensively investigated in the free-living nematode Caenorhabditis elegans [6, 7], which is arguably the best-characterised and most tractable metazoan model organism [8,9]. Particularly well studied are pathways governing/regulating growth, development, reproduction and lifespan [10-14], including 
those of the canonical "dauer signalling" complex [15-19]. Here, environmental signals are received by chemosensory neurons through the cyclic guanosine monophosphate (cGMP) signalling pathway [15], then transduced via the transforming growth factor- $\beta$ (TGF- $\beta$ ) signalling and insulin/insulin-like growth factor 1 (IGF-1) signalling pathways in neuroendocrine tissues $[17,18]$, and converted to steroid hormone signals (e.g. dafachronic acids, DAs) to regulate cellular processes critical to development, reproduction and lifespan $[10,11,19,20]$. Although homologous signalling pathways have been proposed or partially described for parasitic nematodes [21-26], knowledge is incomplete and functional information mostly lacking. Clearly, the availability of comprehensive genomic and transcriptomic data sets for nematodes of the order Strongylida (parasite.wormbase.org) [27-31], which belong to the same evolutionary clade as C. elegans, provides a unique opportunity and foundation to explore the dauer signalling pathway in socioeconomically important representatives of this order.

Haemonchus contortus (barber's pole worm) is one such representative, arguably one of the most important gastrointestinal nematodes of ruminants worldwide [32]. This worm has a complex life-cycle from the egg to the adult stage, under variable environmental and host conditions [33]. Specifically, the third-stage larvae (L3s) of $H$. contortus survive in the environment and will not develop to the next stage until activated by cues in the gut of the host animal; similarly, the fourth-stage larvae (L4s) may arrest in development within the host in response to seasonal and/or host factors [34-36]. Although previous studies have explored aspects of TGF- $\beta$ and IGF-1 signalling pathways in $H$. contortus and investigated the functionality of selected molecules, including Hc-hsp-90, Hc-daf-16 and Hc-daf-2 [22, 37-44], there are still significant gaps in our knowledge and understanding of the signalling pathways linked to dauer in this species.

Here, employing extensive, publicly available genomic and transcriptomic datasets for $H$. contortus, and guided by molecular and functional resources available for $C$. elegans, we infer the gene homologues representing four inter-connected (dauer-associated) signalling pathways in $H$. contortus and explore developmental transcriptomic, proteomic and phosphoproteomic profiles for pathway components. This pathway reconstruction and validation should provide a basis for functional studies of dauer signalling in $H$. contortus and related nematodes, and might facilitate the discovery of new anthelmintic targets.

\section{Methods}

Identifying $C$. elegans gene homologues in $H$. contortus

A list of all genes $(n=102)$ and gene products $(n=182)$ representing the cGMP, TGF- $\beta$ and IGF-1 signalling pathways as well as the steroid hormone pathway in $C$. elegans was established based on published information [16, 39, 45] (Additional file 1: Table S1). The gene and protein sequences, their accession numbers and transcriptomic data were obtained from WormBase (v.WS261). Homologues of these genes were identified by searching (tblastn; $e$-value: $\leq 10^{-5}$ ) the C. elegans protein sequences against gene predictions from the latest, published genome and the transcriptomes of $H$. contortus [28-31]. The C. elegans protein sequences were also searched against the $H$. contortus genome using BLAT v.34 [46] to identify homologues. The gene sequences identified were compared (blastx; $e$-value: $\leq 10^{-5}$ ) with C. elegans proteins (PRJNA13758.WS261) to cross-verify their identity.

\section{Gene curation and structural modelling}

Genes and transcripts were curated using a recently established method [47]. In brief, the sequences inferred to represent homologues were mapped to the genome assembly of $H$. contortus using the program BLAT v.34; mapping was displayed using the Integrated Genome Viewer v.2.4.4 (IGV). The mapped transcripts were reassembled using the program CAP3 [48] for possible extensions. The reassembled transcript sequences were mapped to the genome assembly of $H$. contortus [29], and the corresponding coding DNA sequences (CDS) in the genome were refined using the "coding2genome" model in the program Exonerate v.2.2.0 [49]. The sequences of curated genes were cross-checked with those of complementary DNAs (cDNAs) for Hc-daf-16, Hc-daf-2 and $H c-p d k-1$ [41, 43, 44]. Subsequently, open reading frames (ORFs) were predicted using the program ORF finder [50], and structural and functional domains identified using InterProScan v.61.0 [51, 52]. Pairwise comparisons of inferred amino acid sequences were performed using the program MAFFT v.7.309 [53].

Structural modelling was conducted for a nuclear hormone receptor (DAF-12) using the program I-TASSER [54], following the alignment of amino acid sequence data in MAFFT v.7.309. The alignment was viewed in MView v.1.62 [55]. Models were displayed and compared with available crystal structures [56] using UCSF Chimera v.1.12 [57], and structural similarities between query and template sequences were measured using TM-score and root-mean-square deviation (RMSD) [54]. Biological functions (Gene Ontology, GO) of the modelled protein domain were inferred based on structural similarity.

\section{Transcription analysis}

RNA-seq reads (paired-end) from individual developmental stages/sexes of the nematode were mapped to individual curated CDS in the genome using Bowtie 
v.2.1.0 within the software package RSEM v.1.2.11 [58, 59]. At least 10 reads needed to map to a CDS for transcription to be recorded. Transcription levels of messenger RNAs (mRNAs) were recorded in fragments per kilobase per million mapped reads (FPKM). For individual genes of individual developmental stages of $H$. contortus, transcription levels were displayed in a heat map using heatmap.2 in an R-language environment (v.3.5.1).

\section{Protein analyses}

Proteomic analysis of $H$. contortus was conducted using an established protocol [31]. In brief, protein sequences predicted from individual homologous genes were used to search mass spectrometric (MS) data representing the egg, L3, L4 (female and male) and adult (female and male) stages of $H$. contortus using Proteome Discoverer software v.2.0 (Thermo Fisher Scientific, San Jose, CA, USA). Peptides were identified using a false discovery rate (FDR) cut-off of $<1 \%$ at the peptide and protein levels. Peptide intensities were calculated using Spectronaut software v.11 (Biognosys). At least two peptides needed to match a corresponding protein sequence for expression to be recorded. Peptide intensities were employed to infer the expression levels of individual protein homologues in different developmental stages of $H$. contortus. A phosphoproteomic analysis of egg, L3, L4 (female and male) and adult (female and male) stages of $H$. contortus was conducted using an established $\mathrm{TiO}_{2}$ enrichment protocol $[60,61]$. Protein sequences encoded by dauer signalling gene homologues were employed to interrogate the phosphoproteomic data using the Proteome Discoverer software. Phosphopeptides were identified using a FDR cut-off of $<1 \%$ at the peptide and protein levels. Phosphorylated proteins were mapped to dauer signalling pathways in $H$. contortus.

\section{Results}

\section{The dauer signalling pathway model}

First, we identified the genes $(n=102$; Additional file 1: Table S1) representing the cGMP, TGF- $\beta$, IGF- 1 and steroid hormone signalling pathways in C. elegans, and constructed the canonical framework for the dauer signalling pathway model (Fig 1).

Secondly, we identified all homologues of these genes in $H$. contortus. To do this, we conducted exhaustive homology searches against the genome and combined transcriptome of $H$. contortus, which yielded more than 25,000 sequences in $H$. contortus, from which we predicted genes and transcripts. These genes and transcripts, relating to 61 full-length coding sequences, were curated and assigned names (Additional file 1: Table S2). $H c-d a f-1$ was represented by two paralogues (Additional file 1: Table S2). All 61 genes were transcribed, and transcripts had domain sequence-matches with respective amino acid sequences encoded by $C$. elegans genes (Additional file 1: Table S2).

Thirdly, we mapped the 61 genes to the framework constructed for C. elegans (Fig. 1), providing a model for dauer signalling pathways in $H$. contortus. From this mapping, 60 of the 102 C. elegans dauer signalling genes were homologous to the 61 genes in $H$. contortus. However, not detected in the parasitic nematode were genes encoding proteins SRBC-64, SRBC-66, SRG-36 and SRG37 (G protein-coupled receptors, GPCRs); AKT-2 (serine/threonine kinase), PAR-5 (14-3-3 protein), DAF-28 (insulin), INS-2 to INS-16 and INS-19 to INS-37 (insulin-like peptides); and NCR-2 (membrane permease), inferred or known to be involved in dauer signalling in C. elegans.

\section{Signalling cascade}

Based on our model for $H$. contortus, we hypothesise the following events in the four components of the signalling cascade:

For cGMP signalling (8 components; Additional file 1: Table S2), environmental cues are sensed by $\mathrm{Hc}$-DAF-37 and $\mathrm{Hc}$-DAF-38 (GPCRs) that activate $\mathrm{Hc}$-GPA-2 and $\mathrm{Hc}$ GPA-3 (GTPase), which initiates cGMP production by $\mathrm{Hc}$ DAF-11 (receptor-like guanylyl cyclase) and Hc-DAF-21 (heat shock protein 90), controlling the activity of the transmembrane channels formed by $\mathrm{Hc}$-TAX-2 and $\mathrm{Hc}$ TAX-4 (cGMP-gated channel subunits).

For TGF- $\beta$ signalling (14 components; Additional file 1: Table S2), Hc-DAF-7 (TGF- $\beta$ ) is bound by Hc-DAF-1 and $H c$-DAF-4 (heterotetrameric TGF- $\beta$ receptor), which activates $H c$-DAF- 8 and $H c$-DAF-14 (Smad protein complex) that is inhibited by $H c$-PDP-1 (serine/threonine phosphatase), inhibiting the function of $\mathrm{Hc}$-DAF-3 and Hc-DAF-5 (transcription regulator complex) which are regulated by $\mathrm{Hc}$-EGL-4 (cGMP-dependent protein kinase), $\mathrm{Hc}$-SCD-1 and Hc-SCD-2 (receptor tyrosine kinases) and $H c$-FSN-1 (F-box synaptic protein).

For IGF-1 signalling (26 components; Additional file 1: Table S2), Hc-ASNA-1 (ATPase) promotes the secretion of $H c$-INS-1, $H c$-INS-17 and Hc-INS-18 (insulin-like peptides) from chemosensory neurons, which bind to $H c$-DAF-2 and Hc-IST-1 (receptor tyrosine kinase and substrate) that regulate sequential phosphorylation by Hc-AAP-1, Hc-AGE-1 (phosphoinositide 3-kinase), $\mathrm{Hc}$ PITP-1 (phosphatidylinositol transfer protein), Hc-PDK-1 (3-phosphoinositide-dependent kinase), $\mathrm{Hc}$-SGK-1 and $\mathrm{Hc}$ AKT-1 (serine/threonine kinases), regulating the cytoplasmic sequestration of transcription factors $\mathrm{Hc}$-HSF-1 (with Hc-DDL-1 and heat shock binding protein Hc-HSB-1), $H c$-SKN-1 and Hc-DAF-16 (with 14-3-3 protein FTT-2). 


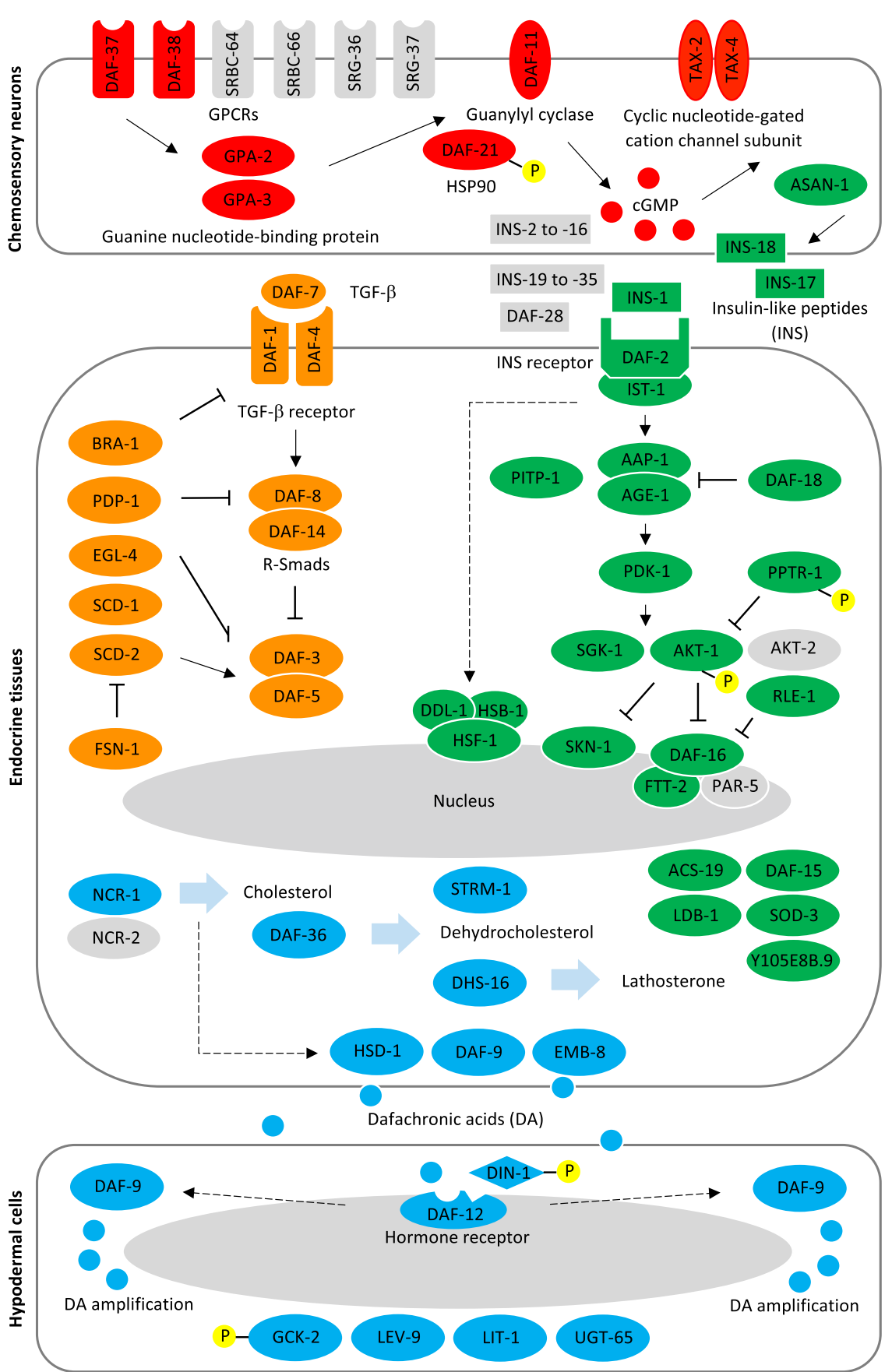

Fig. 1 Dauer signalling pathway model proposed for Haemonchus contortus. The CGMP (red), TGF- $\beta$ (orange), insulin-like growth factor 1 (IGF-1) (green) and steroid hormone (light blue) signalling pathways are constructed based on information and data available for C. elegans. Pathway components not identified in $\mathrm{H}$. contortus are indicated in grey. It is hypothesized that environmental signals are received by G-protein-coupled receptors (GPCRs) through cGMP signalling in chemosensory neurons, transduced via TGF- $\beta$ and insulin-like peptides in parallel TGF- $\beta$ signalling and IGF-1 signalling, which then converge into the steroid hormone signalling pathway via the biosynthesis of dafachronic acids (DAs; ligands of nuclear hormone receptor DAF-12) in neuroendocrine tissues, determining the activation of DAF-12 in target (hypodermal) cells. The activation of DAF-12 initiates or regulates numerous molecular events such as transcription. Signalling or regulation is indicated by an arrow or cross-line. Phosphorylation events detected in the third larval stage (L3) of H. contortus are indicated by a phosphate group (yellow). Dashed arrows indicate pathways which need to be further elucidated. Drawing is adapted or modified from previous publications $[24,39,45,62]$ 
Hc-DAF-18 (lipid phosphatase), Hc-PPTR-1 (serine/threonine phosphatase) and $H c$-RLE-1 (E3 ubiquitin ligase) regulate the phosphorylation of $\mathrm{Hc}$-AGE-1, Hc-AKT-1 and Hc-DAF-16, respectively. The interactors in this signalling pathway also include $H c$-ACS-19 (acyl-CoA synthetase), $H c$-DAF-15 (raptor protein), Hc-LDB-1 (LIM Domain Binding protein), $\mathrm{Hc}$-SOD-3 (iron/manganese superoxide dismutase) and a homologue of Y105E8B.9 (glutathione transferase).

For steroid hormone signalling (13 components; Additional file 1: Table S2), extracellular cholesterol and glycolipids are transported into neuroendocrine cells by Hc-NCR-1 (membrane permease), and sequentially modified by Hc-DAF-36 (Rieske oxygenase), Hc-STRM-1 (methyltransferase), $H c$-DHS-16 (dehydrogenase), $H c$-DAF-9 (cytochrome P450) and Hc-EMB-8 (NADPH-cytochrome P450 reductase), or directly modified by an Hc-HSD (hydroxysteroid dehydrogenase), resulting in the production of DAs that activate $H c$-DAF-12. In turn, the activation of $\mathrm{Hc}$-DAF-12 promotes the production of DAs via $\mathrm{Hc}$-DAF-9 in target (hypodermal) cells. The binding of DA or $H c$-DIN-1 (nucleic acid binding protein) to $H c-D A F-12$ (nuclear hormone receptor) forms a co-activator or corepressor complex, regulating downstream Hc-GCK-2 (mitogen-activated protein kinase kinase kinase kinase), Hc-LEV-9 (sushi domain-containing protein), Hc-LIT-1 (serine threonine protein kinase) and Hc-UGT-65 (UDP glucuronosyltransferase).

\section{Molecular profiles throughout development}

We established the transcription profiles of dauer signalling genes for all key developmental stages of $H$. contortus (egg, the first-larval (L1), second-larval (L2), L3, L4 stages, and female and male adult stages) and compared these profiles with those inferred for homologues in respective stages of $C$. elegans. In both nematode species, each homologous gene was transcribed in at least one developmental stage. Transcription profiles were similar between the L3 stage of $H$. contortus and dauer stage of C. elegans (Fig 2). In these developmental stages of the two species, peak transcription levels were detected for genes daf-37, gpa-2, gpa-3, daf-11 and tax-2 in the cGMP signalling pathway; daf-14 and egl-4 in the TGF- $\beta$ signalling pathway; ins-1, ins17, ins-18, ist-1, akt-1, skn-1 and daf-16 in the IGF-1 signalling pathway; and $e m b-8$, daf-12 and din- 1 in the steroid hormone signalling pathway (Fig. 2; Additional file 1: Tables S1 and S3). By contrast, there were distinct differences in profiles for other genes. Although high transcription levels were recorded for $d a f-38$ and tax-4
(cGMP signalling), daf-7, daf-1, daf-4, daf-3 and daf-5 (TGF- $\beta$ signalling), daf-2 and pitp-1 (IGF-1 signalling), and $d a f-9$ and $l e v-9$ (steroid hormone signalling) in $H$. contortus L3, transcriptional peaks for these genes were found in L1 or L2 stages of C. elegans (Fig. 2; Additional file 1: Tables S1 and S3). Marked differences in the transcription of selected genes, including daf-21, daf-1, $d a f-4$ and $d a f-2$, were found between the two species in the egg, L4 and adult stages (Fig. 2).

We also explored the protein expression of the dauer signalling genes in $H$. contortus as well as the phosphorylation of proteins. In total, proteins encoded by 11 signalling genes were detected in the egg, L3, L4 and/or adult stages of the parasite (Additional file 1: Table S4). These genes included Hc-daf-21 (cGMP signalling), Hc-egl-4 and $H c-p d p-1$ (TGF- $\beta$ signalling pathway), Hc-akt-1, Hc-pptr-1, Hc-asna-1, Hc-ftt-2 and Hc-sod-3 (IGF-1 signalling), and Hc-emb-8,Hc-din-1 and Hc-gck-2 (steroid hormone signalling). In these proteins, 1 to 6 phosphorylation sites was/were detected for $\mathrm{Hc}$-DAF-21 (cGMP signalling), $H c$-AKT-1 and $H c$-PPTR-1 (IGF-1 signalling), Hc-DIN-1 and Hc-GCK-2 (steroid hormone signalling) (Additional file 1: Table S4). These five phosphorylated proteins were detected in egg and/or larval stages of $H$. contortus, particularly L3. However, none of them was found to be phosphorylated in female or male adult stages of this species.

\section{DAF-12-the checkpoint in dauer signalling}

As DAF-12 is a determining point in the dauer signalling pathway, we explored its features and conservation. Pairwise comparisons of the inferred DAF-12 protein sequences of $H$. contortus (Hc-DAF-12a), C. elegans (WormBase ID: CE27585), Strongyloides stercoralis (GenBank: AAD37372), Ancylostoma ceylanicum (GenBank: EPB79655) and Necator americanus (GenBank: XP_013307217) revealed sequence identities of $35.5 \%$ to $86.3 \%$. High sequence similarities were in the zinc finger (nuclear hormone receptor type) and ligand binding domains (Fig. 3a). The three-dimensional models for the DAF-12 ligand binding domains of $H$. contortus (3.1 $\pm 2.2 \AA)$ and $C$. elegans $(3.1 \pm 2.2 \AA)$ both showed high structural similarity (TM-score: $\geq 0.97)$ to the published crystal structure for DAF-12 (ligand-binding domain 3GYU_chainA; S. stercoralis) (Fig 3b). Based on sequence and structural similarities, the biological processes for Hc-DAF-12 were predicted as DNA-templated regulation of transcription (GO:0006355), steroid hormonemediated signalling pathway (GO:0043401) and ecdysone receptor-mediated signalling pathway (GO:0035076), with $\mathrm{GO}$-scores of $0.94,0.94$ and 0.67 , respectively. 

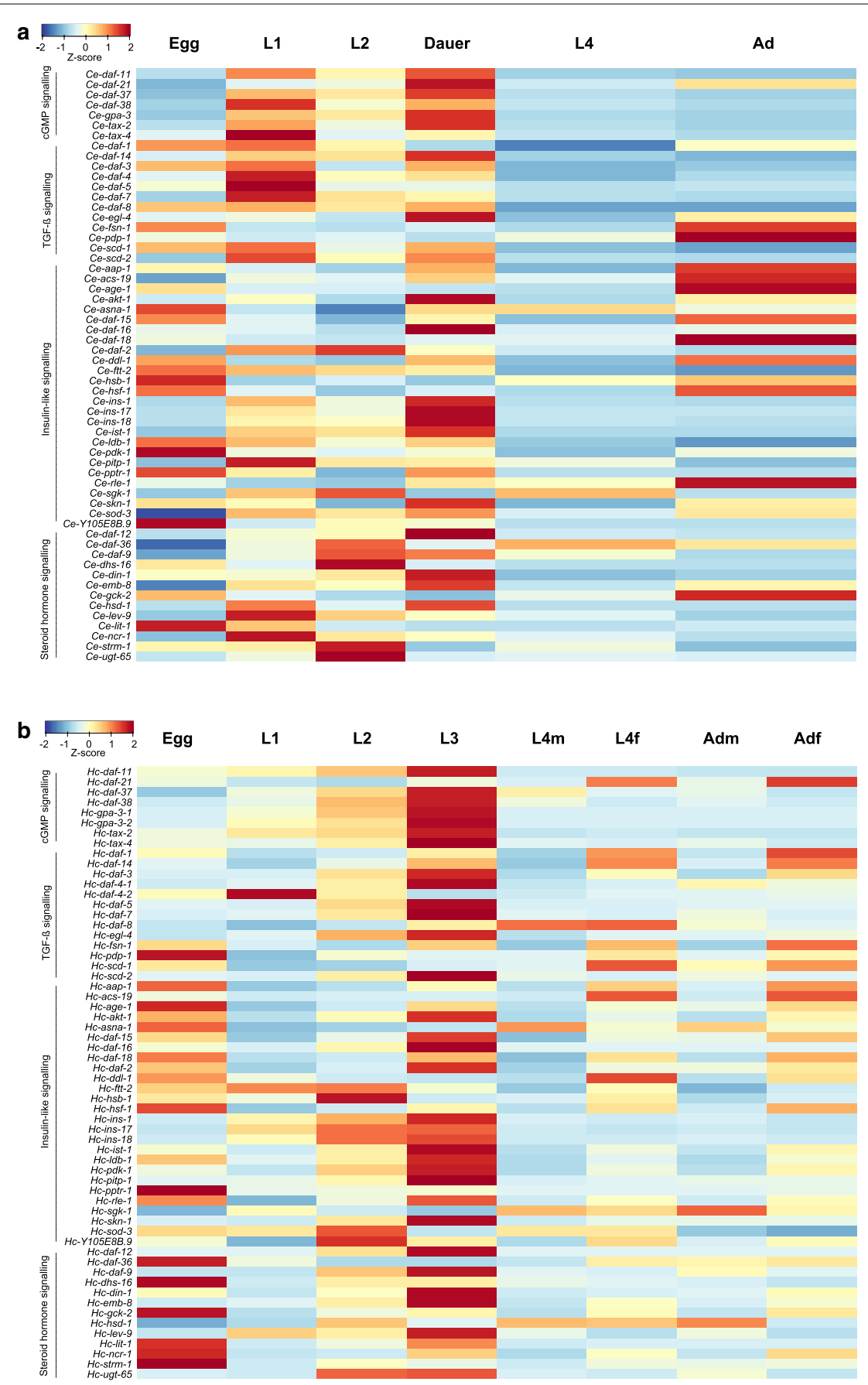

Fig. 2 Transcription of dauer signalling genes in Caenorhabditis elegans and in Haemonchus contortus. Messenger RNA transcriptional levels (fragments per kilobase per million mapped reads, FPKM) of a 60 dauer signalling genes in key developmental stages of $C$. elegans [egg, the first- (L1), second- (L2), dauer-, fourth- (L4) stage larvae and adults (Ad)], and b all 61 homologous genes in H. contortus stages (egg, L1, L2, L3, $\mathrm{L} 4$ (female and male $\mathrm{L} 4 \mathrm{~s}-\mathrm{L} 4 \mathrm{f}$ and $\mathrm{L} 4 \mathrm{~m}$ ), and female and male adults—Adf and Adm) are indicated in the heat maps. Colour scales and Z-scores indicate scaled FPKMs in the rows. Genes involved in the CGMP, TGF- $\beta$, IGF-1 and steroid hormone signalling pathways are listed

\section{Discussion}

Using the enhanced genome and comprehensive transcriptome data sets for $H$. contortus as well as extensive information and data sets available for the related freeliving nematode, $C$. elegans, we constructed a model for all four dauer signalling pathway components in $H$. 


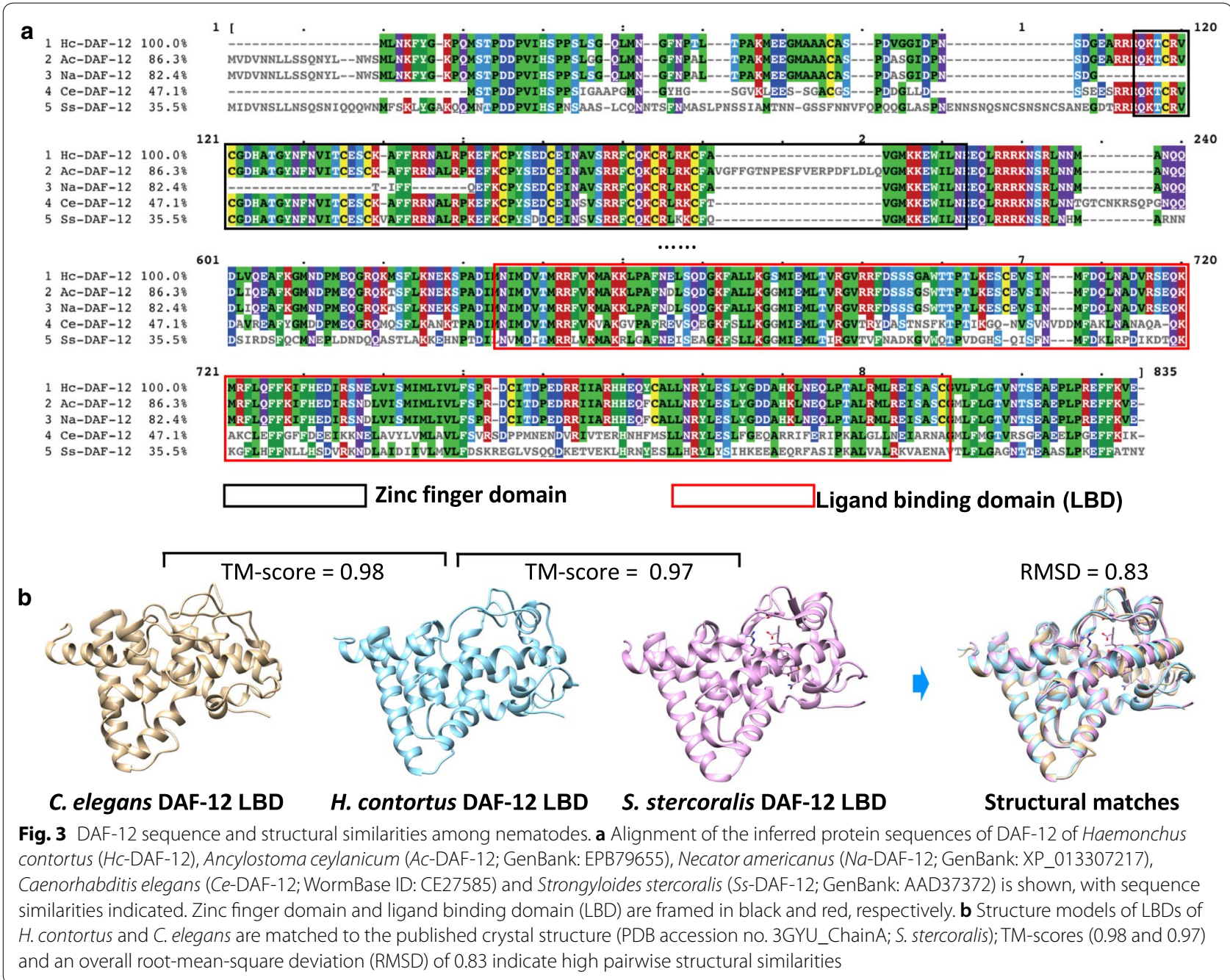

contortus (Fig. 1). Based on this model, we explored gene transcription, expression and phosphorylation of the signalling molecules in all key developmental stages of $H$. contortus, compared the transcriptional profiles with those of stages in C. elegans, and examined the structural and likely functional similarities in a key determining point of the dauer signalling pathway, DAF-12, between these two nematodes. We believe that our model provides a basis to test hypotheses regarding the functions of these signalling pathway components in $H$. contortus, to elucidate the developmental biology of this and related nematodes, and to facilitate the discovery of new anthelmintic targets.

The identification of homologous GPCRs, ligands and receptors, kinases and phosphatases, transcriptional factors and a nuclear hormone receptor (DAF-12) in $H$. contortus implies relative conservation of dauer signalling, a statement which is, to some extent, supported by functional data for genes including age-1, aap-1, daf-2, daf-7, daf-21, daf-16 and pdk-1 in H. contortus [40-44, 63]. Specifically, previous genetic complementation studies have shown that Hc-daf-2, Hc-daf-16 and Hc-daf-21 could at least partially rescue respective null mutants in C. elegans $[40,41,43]$, indicating functionality in the regulation of developmental processes in $H$. contortus.

Although similarities in dauer signalling have been proposed $[21-25,64]$ and verified through in silico studies $[39,45]$, there are some differences in the number of signalling homologues between $C$. elegans and parasitic nematodes. In the present study, although genes coding for pheromone receptors (daf-37 and $d a f-38$ ) and insulinlike peptides (i.e. ins-1, ins-17 and ins-18) that promote dauer formation [18, 60-67] were identified in $H$. contortus, homologues coding for insulin-like peptides (e.g. daf28, ins-4 and ins-6) that promote larval development [18, 68 ] were not detected. Such variation in the number of genes encoding signalling molecules (particular GPCRs, GTPase, insulin-like peptides, serine/threonine kinase 
and membrane permease) has been reported in previous studies of parasitic nematodes including Ascaris suum, $N$. americanus and S. stercoralis [39, 45]. For example, S. stercoralis has only a small number of genes encoding insulin-like peptides, but has an expanded set of TGF$\beta$-coding genes [45]. Although gene predictions depend significantly on the quality of the draft genomes used and bioinformatic algorithms applied [69, 70], the differences seen here are likely explained by the divergent evolution of nematodes, reflecting the marked biological differences among nematodes, both free-living and parasitic $[14,24,26,39]$. Understanding the impact of evolutionary and biological divergences requires detailed studies of a broader range of nematodes species and functional assessments of particular dauer signalling components in them.

The dauer signalling pathway in $H$. contortus appears to play a role in larval diapause. The similar transcriptional patterns of signalling molecules between the infective L3 stage of $H$. contortus and the dauer stage of $C$. elegans indicate a similar function. The similarity in the arrested developmental state between the infective larva (L3) of strongylid nematodes and the dauer form in $C$. elegans has been discussed extensively [21, 22, 71], and the hypobiotic stage was proposed to be a pre-adaptation to parasitism (i.e. dauer hypothesis) [24], although we hasten to add that the L4 stage of some species of parasitic nematodes can undergo hypobiosis in host tissues and seems to be commensurate with dauer [35, 72-74]. This dauer hypothesis is supported by transcriptomic and proteomic evidence from the present study. Specifically, the transcriptional peaks of $d a f-37$ and $d a f-38$, daf-7, ins1, ins-17 and ins-18, and daf-16 in the L3 stage suggest a role in developmental arrest, whereas no translation and high transcription of genes daf-9, daf-12 and din-1 imply an adaptation (e.g. pre-transcription and storage of RNA) for the subsequent parasitic stage of $H$. contortus. Interestingly, the transcription of daf-7 peaks in L3s of $H$. contortus (clade $\mathrm{V}$ ) and of parasitic nematodes representing other clades, including S. stercoralis (clade IV) and Brugia malayi (clade III); the transcription profiles in these nematodes is distinct from the free-living nematode C. elegans (clade V), suggesting a "repurposing" of roles from signalling to other biological processes, such as immunomodulation, in parasitic nematodes [24, 25].

The roles of GPCRs, TGF- $\beta$, insulin-like ligands and fork head transcription factor (i.e. DAF-16) in dauer formation, and cytochrome P450 and nuclear hormone receptor (i.e. DAF-12) in regulating dauer formation in C. elegans have been reported previously [10, 65-67, 75]. However, there is a dearth of information on the functional roles of dauer signalling pathway components in developmental diapause of $H$. contortus [37].
Some explorations of parasitic nematodes, including $A$. caninum and S. stercoralis [76-79], provide functional evidence for cGMP and IGF-1 signalling, and particularly for steroid hormone (DA) signalling, in regulating the activation of infective larvae. Other studies have suggested roles of signalling pathways in hypobiosis (at the L4 stage) in $H$. contortus $[37,80]$ and related nematodes including Ostertagia ostertagi, Teladorsagia circumcincta and Dictyocaulus viviparus [34, 74, 81-84]. Moreover, the different transcriptional profiles of signalling genes in L4 and/or adult stages between $H$. contortus and C. elegans might relate to variation in sexual differentiation between these species. Divergent transcription profiles of signalling molecules have also been reported for nematodes such as Heterodera glycines, Heligmosomoides polygyrus and S. stercoralis [45, $63,85]$, suggesting biological divergence in larval diapause and reproductive development among nematode species. For example, two orphan genes Hc-daf-22 and $H c-f a u$ have been inferred to regulate larval diapause in $H$. contortus [86, 87]. Clearly, functional analyses of signalling cascade components and their regulation are required to improve our understanding of molecular pathways governing developmental processes in parasitic nematodes.

The structural and functional similarities of DAF-12 among free-living and parasitic nematodes suggest a common theme (i.e. a hormone-signalling module) in developmental regulation $[64,88]$. Specifically, the central roles of steroids in the development of nematodes have been extensively reported and discussed, particularly the roles of DA-DAF-12 in regulating a connected network of genes [19, 20,62, 89-91]. Therefore, as a central point in the dauer signalling pathway, DAF-12 has been proposed as a therapeutic target in parasitic nematodes [56, 92, 93]. In addition, non-coding small RNAs might play roles in regulating signalling pathways in $H$. contortus [31]. For example, although substantial transcription of Hc-daf-9 and Hc-daf-12 (i.e. genes involved in promoting reproductive development) [10, 94] was detected in the L3 stage of $H$. contortus, the functions of these genes might be suppressed based on evidence of limited gene translation in this stage. In addition, the marked discrepancies in transcription and expression for genes such as $\mathrm{Hc}$-daf-21, Hc-daf-7 and Hc-emb-8 might be explained by post-transcriptional regulation through microRNAs (miRNAs) [95, 96], as tight regulation by such RNAs has been inferred recently for $H$. contortus [31]. Nonetheless, our understanding of the biological roles of miRNAs in $H$. contortus is in its infancy [31, 97], which contrasts the situation for C. elegans where the regulatory roles of miRNAs in development are well studied [98, 99]. For 
instance, it has been reported that a complex feedback circuit between daf-12 and the let-7 family miRNAs regulates the division of epidermal stem cells during larval development $[13,75]$.

In addition to post-transcriptional regulation, posttranslational modifications are also likely involved in dauer signalling in $H$. contortus. This statement is supported by the present phosphoproteomic results. Phosphorylation was detected on $\mathrm{Hc}$-DAF-21, $\mathrm{Hc}$ PPTR-1, Hc-AKT-1, Hc-DIN-1 and Hc-GCK-2 in the L3 stage of $H$. contortus, indicating an activation of cGMP, insulin-like and steroid hormone signalling pathways. Specifically, the exclusive phosphorylation of $H c$-DAF-21, Hc-DIN-1 and Hc-GCK-2 in L3s suggests roles in arrested development. For C. elegans, it has been reported that phosphorylated PPTR-1 might activate the function of DAF-16 by reducing the inhibition of AKT-1, and that phosphorylated DIN-1 can inhibit the function of DAF-12 by forming a repression complex, promoting dauer diapause in this free-living nematode $[12,18]$. The identification of a range of kinases and their functional assessment in $H$. contortus also indicate extensive phosphorylation during signal transduction and integration and encourage future explorations of nematode tissues to better understand the roles of miRNAs, phosphorylation and lipids in signal transduction events and to discover novel anthelmintic targets. Clearly, available genetic technologies, 'omic resources, informatic tools and hypotheses should facilitate such investigations in $H$. contortus and related parasitic worms.

\section{Conclusions}

Here, we propose a model for the canonical (dauerassociated) cGMP, TGF- $\beta$, IGF-1 and steroid hormone signalling pathways from genomic and transcriptomic data sets for $H$. contortus, guided by resources and data available for C. elegans. This model should provide a basis to test hypotheses pertaining to these pathways and a platform for future functional explorations of molecular biological processes and associated regulatory mechanisms (via miRNAs, phosphorylations and/ or lipids) involved in the development and reproduction of $H$. contortus and related nematodes as well as parasite-host cross-talk, which might guide the discovery of novel therapeutic targets. Although the focus of the present paper was on $H$. contortus, the approach employed for pathway reconstruction could be applied to other nematodes that are relatively closely related to C. elegans, including, for instance, Ostertagia and Trichostrongylus (clade V), and Ascaris and Toxocara (clade III).

\section{Additional file}

Additional file 1: Table S1. Salient information and data for genes known or inferred to be involved in the dauer signalling pathway in Caenorhabditis elegans. Table S2. Dauer signalling gene homologues in Haemonchus contortus. Table S3. Gene transcription of dauer signalling gene homologues in key developmental stages of Haemonchus contortus. Table S4. Protein expression and phosphorylation of dauer signalling gene homologues in key developmental stages of Haemonchus contortus.

\section{Abbreviations}

cGMP: chemosensory neurons through the cyclic guanosine monophosphate; TGF- $\beta$ : transforming growth factor- $\beta$; IGF-1: insulin/insulin-like growth factor 1 ; DAs: dafachronic acids; MS: mass spectrometry.

\section{Acknowledgements}

We are grateful to Dr Ching-Seng Ang, Associate Professor Nicholas A. Williamson and Professor Gavin E. Reid for contributions to published proteomic investigations of $\mathrm{H}$. contortus, and to Professors James B. Lok and David J.

Mangelsdorf for comments on the draft manuscript.

Funding

Funding from the Australian Research Council (ARC), the National Health and Medical Research Council (NHMRC) of Australia and Melbourne Water Corporation and The University of Melbourne (BIP) is gratefully acknowledged (RBG et al.). PKK holds an NHMRC Early Career Research Fellowship. NDY holds an NHMRC Career Development Fellowship.

\section{Availability of data and materials}

Data supporting the conclusions of this article are included within the article. Nucleotide and inferred amino acid sequence data reported in this article are provided in Additional file 1.

\section{Authors' contributions}

GM and TW undertook the laboratory and analytical work. GM, TW and RBG wrote the manuscript, with inputs from PKK, AJS and NDY. All authors read and approved the final manuscript.

Ethics approval and consent to participate Not applicable.

Consent for publication

Not applicable.

Competing interests

The authors declare that they have no competing interests.

\section{Publisher's Note}

Springer Nature remains neutral with regard to jurisdictional claims in published maps and institutional affiliations.

Received: 24 January 2019 Accepted: 28 March 2019

Published online: 29 April 2019

\section{References}

1. Gammons M, Bienz M. Multiprotein complexes governing Wnt signal transduction. Curr Opin Cell Biol. 2017;51:42-9.

2. Gushchin I, Gordeliy V. Transmembrane signal transduction in two-component systems: piston, scissoring, or helical rotation? Bioessays. 2018;40. https://doi.org/10.1002/bies201700197.

3. Deribe $Y L$, Pawson T, Dikic I. Post-translational modifications in signal integration. Nat Struct Mol Biol. 2010;17:666-72.

4. Manning BD, Toker A. AKT/PKB signaling: navigating the network. Cell. 2017; 169:381-405.

5. Hilger D, Masureel M, Kobilka BK. Structure and dynamics of GPCR signaling complexes. Nat Struct Mol Biol. 2018;25:4-12. 
6. Greenwald I. Introduction to signal transduction. WormBook. 2005. http://www.wormbook.org/chapters/www_introsigtrans/introsignaltran sd.html. Accessed 2 Apr 2019.

7. Huang LS, Sternberg PW. Genetic dissection of developmental pathways. WormBook. 2006. https://doi.org/10.1895/wormbook.1.88.2.

8. Corsi AK, Wightman B, Chalfie M. A transparent window into biology: a primer on Caenorhabditis elegans. WormBook. 2015. https://doi. org/10.1895/wormbook.1.177.1.

9. Lee RYN, Howe KL, Harris TW, Arnaboldi V, Cain S, Chan J, et al. WormBase 2017: molting into a new stage. Nucleic Acids Res. 2018;46:D869-74.

10. Gerisch B, Antebi A. Hormonal signals produced by DAF-9/cytochrome $\mathrm{P} 450$ regulate $C$. elegans dauer diapause in response to environmental cues. Development. 2004;131:1765-76.

11. Kenyon C. The plasticity of aging: insights from long-lived mutants. Cell. 2005;120:449-60.

12. Fielenbach $N$, Antebi A. C. elegans dauer formation and the molecular basis of plasticity. Genes Dev. 2007;22:2149-65.

13. Hammell CM, Karp X, Ambros V. A feedback circuit involving let-7-family miRNAs and DAF-12 integrates environmental signals and developmental timing in Caenorhabditis elegans. Proc Natl Acad Sci USA. 2009;106:18668-73.

14. Viney $M$, Harvey S. Reimagining pheromone signalling in the model nematode Caenorhabditis elegans. PLoS Genet. 2017;13:e1007046.

15. Bargmann CL. Chemosensation in C. elegans. WormBook. 2006. https:// doi.org/10.1895/wormbook.1.123.1.

16. Hu PJ. Dauer. WormBook. 2007. https://doi.org/10.1895/wormb ook.1.144.1.

17. Gumienny TL, Savage-Dunn C. TGF- $\beta$ signaling in C. elegans. WormBook. 2013. https://doi.org/10.1895/wormbook.1.22.2.

18. Murphy CT, Hu PJ. Insulin/insulin-like growth factor signaling in C. elegans. WormBook. 2013. https://doi.org/10.1895/wormbook.1.164.1.

19. Antebi A. Nuclear receptor signal transduction in C. elegans. WormBook. 2015. https://doi.org/10.1895/wormbook.1.64.2.

20. Lee SS, Schroeder FC. Steroids as central regulators of organismal development and lifespan. PLoS Biol. 2012;10:e1001307.

21. Tissenbaum HA, Hawdon J, Perregaux M, Hotez P, Guarente L, Ruvkun G. A common muscarinic pathway for diapause recovery in the distantly related nematode species Caenorhabditis elegans and Ancylostoma caninum. Proc Natl Acad Sci USA. 2000;97:460-5.

22. Beall MJ, Pearce EJ. Transforming growth factor-beta and insulin-like signalling pathways in parasitic helminths. Int J Parasitol. 2002;32:399-404.

23. Viney ME, Thompson FJ, Crook M. TGF-beta and the evolution of nematode parasitism. Int J Parasitol. 2005;35:1473-5.

24. Crook M. The dauer hypothesis and the evolution of parasitism: 20 years on and still going strong. Int J Parasitol. 2014;44:1-8.

25. Lok JB. Signaling in parasitic nematodes: physicochemical communication between host and parasite and endogenous molecular transduction pathways governing worm development and survival. Curr Clin Microbiol Rep. 2016;3:186-97.

26. Ma G, Wang T, Korhonen PK, Nie S, Reid GE, Stroehlein AJ, et al. Comparative bioinformatic analysis suggests that specific dauer-like signalling pathway components regulate Toxocara canis development and migration in the mammalian host. Parasit Vectors. 2019:12:32

27. Laing R, Kikuchi T, Martinelli A, Tsai IJ, Beech RN, Redman E, et al. The genome and transcriptome of Haemonchus contortus, a key model parasite for drug and vaccine discovery. Genome Biol. 2013;14:R88.

28. Schwarz EM, Korhonen PK, Campbell BE, Young ND, Jex AR, Jabbar A, et al. The genome and developmental transcriptome of the strongylid nematode Haemonchus contortus. Genome Biol. 2013;14:R89.

29. Doyle SR, Laing R, Bartley DJ, Britton C, Chaudhry U, Gilleard JS, et al. A genome resequencing-based genetic map reveals the recombination landscape of an outbred parasitic nematode in the presence of polyploidy and polyandry. Genome Biol Evol. 2017;10:396-409.

30. Preston S, Korhonen PK, Mouchiroud L, Cornaglia M, McGee SL, Young $N D$, et al. Deguelin exerts potent nematocidal activity via the mitochondrial respiratory chain. FASEB J. 2017:31:4515-32.

31. Ma G, Wang T, Korhonen PK, Ang C-S, Williamson NA, Young ND, et al. Molecular alterations during larval development of Haemonchus contortus in vitro are under tight post-transcriptional control. Int J Parasitol. 2018:48:763-72
32. Gasser RB, von Samson-Himmelstjerna G. Haemonchus contortus and Haemonchosis_-Past, Present and Future. 1st ed. London: Academic; 2016. ISBN: 0128103957

33. Veglia F. The anatomy and life-history of the Haemonchus contortus (Rud). Rep Dir Vet Res. 1915;3-4:347-500.

34. Gibbs HC. Hypobiosis in parasitic nematodes-an update. Adv Parasitol. 1986:25:129-74.

35. Besier RB, Kahn LP, Sargison ND, Van Wyk JA. The pathophysiology, ecology and epidemiology of Haemonchus contortus infection in small ruminants. Adv Parasitol. 2016;93:95-143.

36. Nisbet AJ, Meeusen EN, González JF, Piedrafita DM. Immunity to Haemonchus contortus and vaccine development. Adv Parasitol. 2016;93:353-96.

37. Nikolaou S, Gasser R. Prospects for exploring molecular developmental processes in Haemonchus contortus. Int J Parasitol. 2006;36:859-68.

38. Mohandas N, Hu M, Stroehlein AJ, Young ND, Sternberg PW, Lok JB, et al. Reconstruction of the insulin-like signalling pathway of Haemonchus contortus. Parasit Vectors. 2016;9:64.

39. Gilabert A, Curran DM, Harvey SC, Wasmuth JD. Expanding the view on the evolution of the nematode dauer signalling pathways: refinement through gene gain and pathway co-option. BMC Genomics. 2016;17:476

40. Gillan V, Maitland K, McCormack G, Him NA, Devaney E. Functional genomics of hsp-90 in parasitic and free-living nematodes. Int J Parasitol. 2009;39:1071-81.

41. Hu M, Lok JB, Ranjit N, Massey HC, Sternberg PW, Gasser RB. Structural and functional characterisation of the fork head transcription factorencoding gene, Hc-daf-16, from the parasitic nematode Haemonchus contortus (Strongylida). Int J Parasitol. 2010:40:405-15.

42. Li F-C, Gasser RB, Lok JB, Korhonen PK, Wang Y-F, Yin F-Y, et al. Exploring the role of two interacting phosphoinositide 3-kinases of Haemonchus contortus. Parasit Vectors. 2014;7:498.

43. Li F, Lok JB, Gasser RB, Korhonen PK, Sandeman MR, Shi D, et al. Hc-daf-2 encodes an insulin-like receptor kinase in the barber's pole worm, Haemonchus contortus, and restores partial dauer regulation. Int J Parasitol. 2014;44:485-96.

44. Li F-C, Gasser RB, Lok JB, Korhonen PK, He L, Di W-D, et al. Molecular characterization of the Haemonchus contortus phosphoinositide-dependent protein kinase-1 gene (Hc-pdk-1). Parasit Vectors. 2016;9:65.

45. Stoltzfus JD, Minot S, Berriman M, Nolan TJ, Lok JB. RNAseq analysis of the parasitic nematode Strongyloides stercoralis reveals divergent regulation of canonical dauer pathways. PLoS Negl Trop Dis. 2012:6:e1854.

46. Kent WJ. BLAT-the BLAST-like alignment tool. Genome Res. 2002:12:656-64.

47. Stroehlein AJ, Young ND, Gasser RB. Improved strategy for the curation and classification of kinases, with broad applicability to other eukaryotic protein groups. Sci Rep. 2018;8:6808.

48. Huang X, Madan A. CAP3: a DNA sequence assembly program. Genome Res. 1999:9:868-77.

49. Slater GS, Birney E. Automated generation of heuristics for biological sequence comparison. BMC Bioinformatics. 2005;6:31.

50. Rombel IT, Sykes KF, Rayner S, Johnston SA. ORF-FINDER: a vector for high throughput gene identification. Gene. 2002;282:33-41.

51. Zdobnov EM, Apweiler R. InterProScan - an integration platform for the signature-recognition methods in InterPro. Bioinformatics. 2001;17:847-8.

52. Jones $P$, Binns $D$, Chang HY, Fraser M, Li W, McAnulla C, et al. InterProScan 5: genome-scale protein function classification. Bioinformatics. 2014;30:1236-40.

53. Katoh K, Misawa K, Kuma K, Miyata T. MAFFT: a novel method for rapid multiple sequence alignment based on fast Fourier transform. Nucleic Acids Res. 2002;30:3059-66.

54. Yang J, Yan R, Roy A, Xu D, Poisson J, Zhang Y. The I-TASSER Suite: protein structure and function prediction. Nat Methods. 2015;12:7-8.

55. Brown NP, Leroy C, Sander C. MView: a web compatible database search or multiple alignment viewer. Bioinformatics. 1998;14:380-1.

56. Wang Z, Zhou XE, Motola DL, Gao X, Suino-Powell K, Conneely A, et al. Identification of the nuclear receptor DAF-12 as a therapeutic target in parasitic nematodes. Proc Natl Acad Sci USA. 2009:106:9138-43.

57. Pettersen EF, Goddard TD, Huang CC, Couch GS, Greenblatt DM, Meng EC, et al. UCSF Chimera-a visualization system for exploratory research and analysis. J Comput Chem. 2004;25:1605-12. 
58. Langmead B, Salzberg SL. Fast gapped-read alignment with Bowtie 2. Nat Methods. 2012;9:357-9.

59. Li B, Dewey CN. RSEM: accurate transcript quantification from RNASeq data with or without a reference genome. BMC Bioinformatics. 2011;12:323.

60. Zielinska DF, Gnad F, Jedrusik-Bode M, Wiśniewski JR, Mann M. Caenorhabditis elegans has a phosphoproteome atypical for metazoans that is enriched in developmental and sex determination proteins. J Proteome Res. 2009;8:4039-49.

61. Borchert N, Krug K, Gnad F, Sinha A, Sommer RJ, Macek B. Phosphoproteome of Pristionchus pacificus provides insights into architecture of signaling networks in nematode models. Mol Cell Proteomics. 2012;11:1631-9.

62. Butcher RA. Small-molecule pheromones and hormones controlling nematode development. Nat Chem Biol. 2017;13:577-86.

63. McSorley HJ, Grainger JR, Harcus Y, Murray J, Nisbet AJ, Knox DP, et al. daf7-related TGF-beta homologues from trichostrongyloid nematodes show contrasting life-cycle expression patterns. Parasitology. 2010;137:159-71.

64. Ogawa A, Streit A, Antebi A, Sommer RJ. A conserved endocrine mechanism controls the formation of dauer and infective larvae in nematodes. Curr Biol. 2009;19:67-71

65. Pierce SB, Costa M, Wisotzkey R, Devadhar S, Homburger SA, Buchman $A R$, et al. Regulation of DAF-2 receptor signaling by human insulin and ins-1, a member of the unusually large and diverse C. elegans insulin gene family. Genes Dev. 2001;15:672-86.

66. Matsunaga Y, Gengyo-Ando K, Mitani S, Iwasaki T, Kawano T. Physiological function, expression pattern, and transcriptional regulation of a Caenorhabditis elegans insulin-like peptide INS-18. Biochem Biophys Res Commun. 2012:423:478-83.

67. Matsunaga Y, Nakajima K, Gengyo-Ando K, Mitani S, Iwasaki T, Kawano T. A Caenorhabditis elegans insulin-like peptide, INS-17: its physiological function and expression pattern. Biosci Biotechnol Biochem. 2012;76:2168-72.

68. Li W, Kennedy SG, Ruvkun G. daf-28 encodes a C. elegans insulin superfamily member that is regulated by environmental cues and acts in the DAF-2 signaling pathway. Genes Dev. 2003;17:844-58.

69. Korhonen PK, Young ND, Gasser RB. Making sense of genomes of parasitic worms: tackling bioinformatic challenges. Biotechnol Adv. 2016;34:663-86.

70. Laing R, Martinelli A, Tracey A, Holroyd N, Gilleard JS, Cotton JA. Haemonchus contortus: genome structure, organization and comparative genomics. Adv Parasitol. 2016;93:569-98.

71. Hotez P, Hawdon J, Schad GA. Hookworm larval infectivity, arrest and amphiparatenesis: the Caenorhabditis elegans Daf-c paradigm. Parasitol Today. 1993;9:23-6.

72. Sommerville RI, Davey KG. Diapause in parasitic nematodes: a review. Can J Zool. 2002;80:1817-40.

73. Troell K, Tingstedt C, Höglund J. Phenotypic characterization of Haemonchus contortus: a study of isolates from Sweden and Kenya in experimentally infected sheep. Parasitology. 2006;132:403-9.

74. Strube C, von Samson-Himmelstjerna G, Schnieder T. Genetic regulation of arrested development in nematodes: are age- 1 and daf-gene orthologs present in Dictyocaulus viviparus? Parasitol Res. 2007;101:1111-5.

75. Bethke A, Fielenbach N, Wang Z, Mangelsdorf DJ, Antebi A. Nuclear hormone receptor regulation of microRNAs controls developmental progression. Science. 2009;324:95-8.

76. Hawdon JM, Datu B. The second messenger cyclic GMP mediates activation in Ancylostoma caninum infective larvae. Int J Parasitol. 2003;33:787-93.

77. Datu BJ, Loukas A, Cantacessi C, O'Donoghue P, Gasser RB. Investigation of the regulation of transcriptional changes in Ancylostoma caninum larvae following serum activation, with a focus on the insulin-like signalling pathway. Vet Parasitol. 2009;159:139-48.

78. Stoltzfus JD, Bart SM, Lok JB. CGMP and NHR signaling co-regulate expression of insulin-like peptides and developmental activation of infective larvae in Strongyloides stercoralis. PLoS Pathog. 2014;10:e1004235.

79. Albarqi MM, Stoltzfus JD, Pilgrim AA, Nolan TJ, Wang Z, Kliewer SA, et al. Regulation of life cycle checkpoints and developmental activation of infective larvae in Strongyloides stercoralis by dafachronic acid. PLoS Pathog. 2016;12:e1005358.

80. Kooyman FN, Eysker M. Analysis of proteins related to conditioning for arrested development and differentiation in Haemonchus contortus by two-dimensional gel electrophoresis. Int J Parasitol. 1995;25:561-8.

81. Strube C, Schnieder T, von Samson-Himmelstjerna G. Differential gene expression in hypobiosis-induced and non-induced third-stage larvae of the bovine lungworm Dictyocaulus viviparus. Int J Parasitol. 2007;37:221-31.

82. Dopchiz MC, Parma AE, Fiel CA. Hypobiosis induction alters the protein profile of Ostertagia ostertagi (Nematoda: Trichostrongylidae). Folia Parasitol. 2000;47:135-40

83. Baker RH, Buschbaum S, Matthews JB, McKendrick IJ, Schnieder T, Strube C, et al. GTP-cyclohydrolase and development in Teladorsagia circumcincta and Dictyocaulus viviparus (Nematoda: Strongylida). Exp Parasitol. 2011;128:309-17.

84. Laabs E-M, Schnieder T, Strube C. Transcriptional differences between hypobiotic and non-hypobiotic preadult larvae of the bovine lungworm Dictyocaulus viviparus. Parasitol Res. 2012;110:151-9.

85. Elling AA, Mitreva M, Recknor J, Gai X, Martin J, Maier TR, et al. Divergent evolution of arrested development in the dauer stage of Caenorhabditis elegans and the infective stage of Heterodera glycines. Genome Biol. 2007:8:R211.

86. Yan B, Guo X, Zhou Q, Yang Y, Chen X, Sun W, et al. Hc-fau, a novel gene regulating diapause in the nematode parasite Haemonchus contortus. Int J Parasitol. 2014;44:775-86.

87. Guo X, Zhang H, Zheng X, Zhou Q, Yang Y, Chen X, et al. Structural and functional characterization of a novel gene, Hc-daf-22, from the strongylid nematode Haemonchus contortus. Parasit Vectors. 2016;9:422.

88. Bento G, Ogawa A, Sommer RJ. Co-option of the hormone-signalling module dafachronic acid-DAF-12 in nematode evolution. Nature. 2010;466:494-7.

89. Motola DL, Cummins CL, Rottiers V, Sharma KK, Li T, Li Y, et al. Identification of ligands for DAF-12 that govern dauer formation and reproduction in C. elegans. Cell. 2006;124:1209-23.

90. Hochbaum D, Zhang Y, Stuckenholz C, Labhart P, Alexiadis V, Martin R, et al. DAF-12 regulates a connected network of genes to ensure robust developmental decisions. PLoS Genet. 2011;7:e1002179.

91. Wang Z, Stoltzfus J, You Y-J, Ranjit N, Tang H, Xie Y, et al. The nuclear receptor DAF-12 regulates nutrient metabolism and reproductive growth in nematodes. PLoS Genet. 2015;11:e1005027.

92. Wang Z, Schaffer NE, Kliewer SA, Mangelsdorf DJ. Nuclear receptors: emerging drug targets for parasitic diseases. J Clin Invest. 2017;127:1165-71.

93. Patton JB, Bonne-Année S, Deckman J, Hess JA, Torigian A, Nolan TJ, et al. Methylprednisolone acetate induces, and $\Delta 7$-dafachronic acid suppresses, Strongyloides stercoralis hyperinfection in NSG mice. Proc Natl Acad Sci USA. 2018;115:204-9.

94. Antebi A, Yeh WH, Tait D, Hedgecock EM, Riddle DL. daf-12 encodes a nuclear receptor that regulates the dauer diapause and developmental age in C. elegans. Genes Dev. 2000;14:1512-27.

95. Filipowicz W, Bhattacharyya SN, Sonenberg N. Mechanisms of posttranscriptional regulation by microRNAs: are the answers in sight? Nat Rev Genet. 2008;9:102-14.

96. Bartel DP. MicroRNAs: target recognition and regulatory functions. Cell. 2009;136:215-33

97. Winter AD, Weir W, Hunt M, Berriman M, Gilleard JS, Devaney E, et al. Diversity in parasitic nematode genomes: the microRNAs of Brugia pahangi and Haemonchus contortus are largely novel. BMC Genomics. 2012;13:4.

98. Boehm M, Slack F. A developmental timing microRNA and its target regulate life span in C. elegans. Science. 2005;310:1954-7.

99. Ahmed R, Chang Z, Younis AE, Langnick C, Li N, Chen W, et al. Conserved miRNAs are candidate post-transcriptional regulators of developmental arrest in free-living and parasitic nematodes. Genome Biol Evol. 2013;5:1246-60. 